\title{
Relationship Between AMACR Staining Density of Radical Prostatectomy Specimen and Biochemical Recurrence in Patients with Pathological Stage T2a-b
}

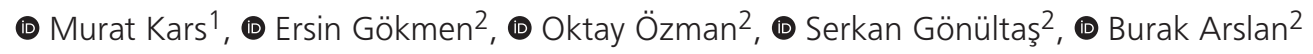 \\ 1 Gebze Medical Park Hospital, Clinic of Urology, Istanbul, Turkey \\ 2 University of Health Sciences, Gaziosmanpaşa Taksim Training and Research Hospital, Clinic of Urology, Istanbul, Turkey
}

\begin{abstract}
Objective: This study aimed to evaluate the relationship between Alpha-methylacyl-CoA racemase (AMACR) staining intensity of pathologic specimens and Prostate Spesific Antigen (PSA) recurrence of patients with an organ-limited disease who underwent radical prostatectomy for localized prostate cancer.

Materials and Methods: The data of 46 patients who underwent radical retropubic prostatectomy and who had prostate-limited disease without capsule invasion in the pathological evaluation were included in the study. AMACR expression was assessed by immunohistochemical staining. Expression levels were classified as negative $($ score $=0)$, weak $($ score $=1)$, moderate $($ score $=2)$ and strong (score $=3$ ) staining. Patients with a PSA value of $\geq 0.2 \mathrm{ng} / \mathrm{mL}$ at postoperative follow-up were considered to have a biochemical recurrence. Patients were divided into two groups, according to biochemical recurrence. Groups were compared in terms of AMACR staining intensities of radical prostatectomy specimens.

Results: Twenty-one patients developed PSA recurrence in a mean follow-up period of $32 \pm 8$ months. The remaining 25 patients were recurrence-free. The mean recurrence time was $24 \pm 5$ months. According to AMACR staining scores, the biochemical recurrence rates of the patients were $33.3 \%$, $40.7 \%$, and $61.5 \%$ for score 1, score 2, and score 3, respectively. Although higher rates of PSA recurrence were observed in patients with more intense AMACR staining, the difference between rates was not statistically significant $(p=0.38)$.

Conclusion: PSA recurrence rate increases with increasing AMAC-CR staining intensity. Further studies are needed to obtain statistically significant results.
\end{abstract}

Keywords: AMACR, PSA, prostate cancer

\section{Introduction}

Alpha-methylacyl-CoA racemase (AMACR) is a protein enzyme that provides beta-oxidation of branched fatty acids. The enzyme localized in mitochondria and peroxisomes is also known as P504S. Peroxisomal oxidation is a potential source of oxidative damage to the cell and causes the accumulation of carcinogenic hydrogen peroxide.

Several studies have shown increased AMACR-mRNA transcription in prostate cancer tissues $(1,2)$. Transcript levels of the enzyme showed an average 3.75-fold increase in prostate cancer tissue when compared to BPH. AMACR was highlighted as one of the few genes explaining pathway dysregulation for prostate cancer in a gene meta-analysis (3). Pathology studies with immunohistochemical staining techniques and biochemical studies have reported similar results (4). Increased transcription of the AMACR gene and increased activity of the enzyme in cancer cells compared to benign prostate tissues suggest that the molecule can be used as a biomarker for prostate cancer. AMACR protein expression levels were first shown to be significantly associated with prostate cancer progression, and the reduced AMACR expression level was associated with

Cite this article as: Kars M, Gökmen E, Özman O, Gönültaş S, Arslan B. Relationship Between AMACR Staining Density of Radical Prostatectomy Specimen and Biochemical Recurrence in Patients with Pathological Stage T2a-b. Bull Urooncol 2020;19(1):38-41 
increased biochemical recurrence and cancer-related death (5). Patients with high Gleason scores and low AMACR expression had an 18-fold higher risk of prostate cancer death.

This study aimed to evaluate the relationship between AMACR staining intensity of pathologic specimens and Prostate Spesific Antigen (PSA) recurrence of patients who underwent radical prostatectomy for the low and intermediate-risk organ-confined disease.

\section{Materials and Methods}

The data of 320 patients who underwent radical retropubic prostatectomy for localized prostate cancer in our clinic were evaluated retrospectively. The following exclusion criteria were taken into consideration;

1- Pathological stage T2c and above

2- Positive lymph node metastasis

3- Preoperative PSA $>20 \mathrm{ng} / \mathrm{mL}$

4- Biopsy Gleason Score $>7$ (ISUP grade group 4-5)

Forty-six patients with organ-confined disease in radical prostatectomy pathology were included in the study (pathological stage T2a and T2b). All of the patients have low and intermediaterisk prostate cancer according to the D'Amico risk classification (Gleason score 6 or 7, PSA $<20 \mathrm{ng} / \mathrm{mL}$ ) (6). Patients with a PSA value of $\geq 0.2 \mathrm{ng} / \mathrm{mL}$ at postoperative follow-up were considered to have a biochemical recurrence. Patients were divided into two groups, according to biochemical recurrence.

All hematoxylin eosin-stained sections of radical prostatectomy specimens were examined. The best samples for the tumor and the most suitable preparations for immunohistochemical examination were selected. Then the appropriate sites for immunostaining were marked. Re-sections were taken from paraffin blocks to precisely sample the marked areas. Deparaffinization and clarification were applied to the sections before immunohistochemical staining. Immunohistochemical staining was performed using Primary Antibody AMACR/P504S (Polyclonal Rabbit Antibody, P504S, Catalog Number: CP 200 AK, BK, CK) and UltraTek Anti-Rabbit (UltraTek HRP Anti-Rabbit (DAB) Staining System, Logan, Utah, USA)

We formed four groups according to staining intensity (Figure 1) (Table 1) (7). After staining, it was seen that the preparations contained areas showing different staining intensity. In order to prevent the preparations from having more than one staining score, the area with the highest staining intensity was scored for the relevant preparation. Furthermore, since all of the evaluated preparations containing areas with negative staining scores simultaneously included one of the other staining scores, no preparation received negative staining scores.

\section{Statistical Analysis}

The chi-square and Fisher's exact tests were used for the difference between the categorical variables. The Mann-Whitney $\mathrm{U}$ and Kruskal-Wallis tests were used for the difference between the means. For more than two groups, the ANOVA test was used. The data were analyzed with the Statistical Package for the Social Sciences v. 17 (SPSS Inc., Illinois, USA). A p value under 0.05 was considered statistically significant.

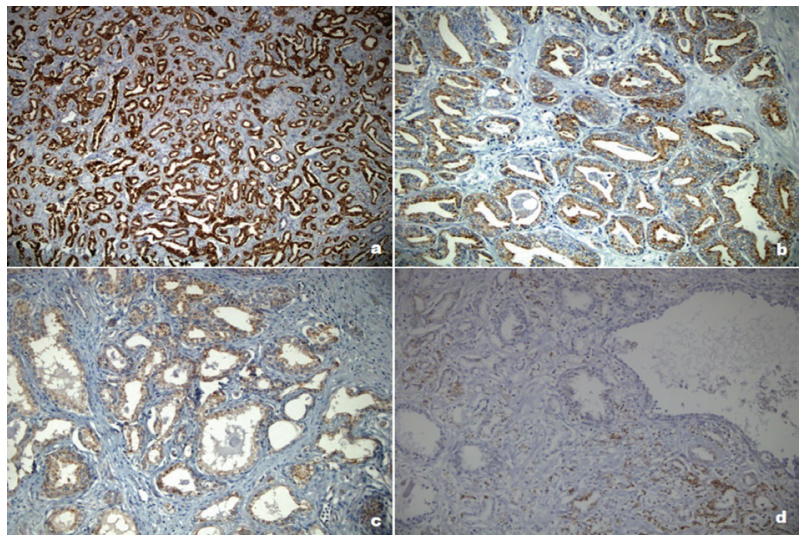

Figure 1. (a) Diffuse, strong cytoplasmic staining (AMACR X 100) in tumor tissue forming 2 abortive gland formation. (b) Disseminated, moderate cytoplasmic staining of tumor tissue 3 forming the abortive gland formation (AMACR X 200). (c) Weak cytoplasmic staining of 4 tumor tissue forming the abortive gland formation (AMACR X 200). (d) Negative staining of 5 tumor tissue forming abortive gland formation and weak cytoplasmic staining in the right 6 lower part (AMACR X 100).

\begin{tabular}{|l|l|}
\hline Table 1. Staining classification of samples \\
\hline Score & AMACR staining \\
\hline 0 & Negative staining \\
\hline 1 & Weak cytoplasmic staining \\
\hline 2 & Disseminated, moderate cytoplasmic staining \\
\hline 3 & Diffuse, strong cytoplasmic staining \\
\hline AMACR: Alpha-methylacyl-CoA racemase \\
\hline
\end{tabular}

\section{Results}

The mean age of 46 patients was $65.23 \pm 6.36$ years. The mean preoperative serum PSA level was $8.96 \pm 6.78 \mathrm{ng} / \mathrm{mL}$, the mean number of positive biopsy quadrants was $2.60 \pm 1.74$, the mean tumor percentage was $13.04 \pm 15.10$, and the prostate volume was $49.89 \pm 19.94$ cc. The mean follow-up period was $32 \pm 8$ (3-61) months. The mean recurrence time was $24 \pm 5$ (3-59) in patients with PSA recurrence.

Twenty-one patients with PSA recurrence and 25 patients without recurrence were compared for preoperative and pathological data. The two groups were similar in terms of age, preoperative serum PSA levels, biopsy Gleason scores, positive number of biopsy quadrants, percentile of tumors, and prostate volume $(\mathrm{p}=0.3, \mathrm{p}=0.8, \mathrm{p}=0.32, \mathrm{p}=0.98, \mathrm{p}=0.47, \mathrm{p}=0.4$ respectively, Table 2).

Biochemical recurrence after radical prostatectomy was observed in $33.3 \%(2 / 6), 40.7 \%(11 / 27)$, and $61.5 \%(8 / 13)$ of patients with AMACR staining intensity score 1, 2 and 3, respectively. As the immunohistochemical staining intensity increased with AMACR, the rate of PSA recurrence increased. However, there was no statistically significant difference between the groups in terms of biochemical recurrence rates $(p=0.377)$ (Table 3$)$.

The relationship between Gleason score, the percentile of tumors, and AMACR immunohistochemical staining intensity were investigated in radical prostatectomy material. There was no statistically significant relationship between Gleason score 


\begin{tabular}{|c|c|c|c|}
\hline Means of characteristic & PSA recurrence + & PSA recurrence - & $\mathbf{p}^{*}$ \\
\hline Age (years) & $64.23 \pm 6.51$ & $66.08 \pm 6.23$ & 0.31 \\
\hline Preoperative PSA (ng/mL) & $9.26 \pm 7.33$ & $8.68 \pm 6.43$ & 0.84 \\
\hline Biopsy Gleason scores & $6.16 \pm 0.37$ & $6.33 \pm 0.48$ & 0.32 \\
\hline Number of positive TRUS-Bx sample & $2.65 \pm 1.93$ & $2.56 \pm 1.60$ & 0.98 \\
\hline Percantile of tumor (\%) & $13.43 \pm 16.92$ & $13.10 \pm 13.75$ & 0.47 \\
\hline Prostate volume (\%) & $59.28 \pm 22.15$ & $47.2 \pm 17.89$ & 0.40 \\
\hline
\end{tabular}

\begin{tabular}{|l|l|l|l|l|}
\hline Table 3. Relationship between AMACR staining score and PSA recurrence & \multicolumn{2}{l|}{ Biopsy Gleason score } \\
\hline Scores & PSA recurrence + & PSA recurrence - & p & $6.17 \pm 0.41$ \\
\hline 1 & $2(33.3 \%)$ & $4(66.7 \%)$ & - & $6.33 \pm 0.48$ \\
\hline 2 & $11(40.7 \%)$ & $16(59.3 \%)$ & $0.38^{*}$ & $6.08 \pm 0.28$ \\
\hline 3 & $8(61.5 \%)$ & $5(38.5 \%)$ & - & $0.19^{\alpha}$ \\
\hline$p$ & - & - & - & \\
\hline $\begin{array}{l}\text { AMACR: Alpha-methylacyl-CoA racemase, PSA: Prostate Spesific Antigen } \\
{ }^{*} \text { Chi-square test, }{ }^{\alpha} \text { ANOVA test }\end{array}$ & & \\
\hline
\end{tabular}

and AMACR staining intensity $(p=0.568)$. Tumor percentages in prostatectomy specimens of patients with AMACR staining intensity score 1, score 2, and score 3 were $14.16 \pm 13.68 \%$, $13.37 \pm 16.60 \%$ and $11.82 \pm 13 \%$, respectively. It was found that the percentage of tumors decreased in the specimens as staining score increased. However, the difference between the groups was not statistically significant $(p=0.97)$.

\section{Discussion}

Rubin et al. (7) have reported no significant relationship between AMACR protein expression and pathological parameters such as radical prostatectomy Gleason score, tumor stage, tumor percentage, and surgical margin positivity. Also, AMACR expression level was not associated with recurrence of PSA following surgery at a mean follow-up of 3 years in a series of 120 patients. In our study, it was found that PSA recurrence rates increased with increasing AMACR expression levels. However, there was no statistically significant difference between the groups. AMACR protein expression was evaluated in two localized prostate cancer groups of 204 patients treated with radical prostatectomy, and 188 patients followed with watchful-waiting in another study conducted by Rubin et al. (5). The development of PSA recurrence in the surgical group and cancer-related death in the follow-up group were considered endpoints, and the AMACR staining intensity was evaluated both manually and using a computer-assisted program. In the surgery-treated group, no significant correlation was found between the AMACR expression score and PSA recurrence, as was the case in previous studies. Small differences in clinical character were observed when comparing low and high AMACR expression in computer-assisted evaluation. In contrast, those with low AMACR expression were found to develop twice as much PSA recurrence during follow-up as compared to those with high expression. The cut-off value of AMACR intensity was 1.11 SD in the surgery group and 0.18 SD in the follow-up group. PSA recurrence was found in $37.5 \%$ of the patients below this cut-off value in the surgery group. This rate was found to be $14.5 \%$ in patients above that value. Patients with lower AMACR expression have a significantly higher risk of developing PSA recurrence. Three-fold more cancer-related deaths were observed in those with AMACR expression below the cut-off value in the follow-up group. Patients with low AMACR expression and high Gleason scores are reported to have a 4-fold increased risk of PSA recurrence and an 18-fold higher risk of cancer-related death.

Intense AMACR staining was reported in untreated metastatic and hormone-resistant prostate cancer cases in a study by Luo et al. (8). The author emphasized that combined staining for p63 and AMACR facilitates the detection of cancer cells in both biopsy and surgical specimens. Studies with the triple stain antibody cocktail obtained by adding high-molecular-weight keratin to these two antibodies are also available in the literature (9). The results of studies using PCR-based detection of AMACR are similar to the results obtained by immunohistochemical staining techniques and provide data that potentiate these results (10). AMACR-mRNA transcription in malignant prostate tissue has been shown to increase several-fold. Kumar-Sinha et al. (10) also reported increased AMACR enzymatic activity in prostate cancer tissues compared to benign prostate tissue.

Molinié et al. (11) emphasize that AMACR staining efficacy may be particularly prominent in suspicious biopsy sites smaller than $1 \mathrm{~mm}$. Diagnosis for atypical small acinar proliferation may become easier with AMACR (12).

In recent trials, AMACR has been the subject of studies to detect prostate cancer by molecular imaging. There are positive results in the literature that molecular imaging is possible using the transcriptional specificity of the AMACR promoter (13). Gene fusions are thought to play a critical role in the development and progression of prostate cancer. Yang et al. (13) found the SLC45A2-AMACR fusion gene in their current study (14). From 
understanding the genetic basis of prostate cancer to easier diagnosis, AMACR is often included basic researches a promising protein. It was shown that patients with biopsy-proven prostate cancer had higher semen AMACR levels than the control group in a study in which semen AMACR levels were measured using the indirect sandwich ELISA chemiluminescence assay (15). Also, AMACR can be used mainly in the treatment of castrationresistant prostate cancer (16).

In our study, there was no significant relationship between AMACR protein expression and PSA recurrence. Unlike Rubin et al. (7), there were no patients with very low or very high Gleason scores in our series (Gleason 5 or 8-9). Also, in our study, no patients were showing surgical margin positivity and extracapsular extension, while patients with these characteristics were present in the mentioned study. In summary, the results of the recent study determined by the patients with both clinically and pathologically localized prostate cancer who were more challenging to predict the development of PSA recurrence. Also, Rubin's study evaluated the AMACR expression intensity both manually and using a computer-assisted program, and indicated that computer-assisted evaluation was more reliable (7).

\section{Study Limitations}

There are some limitations to this study. First of all, direct visual evaluations mentioned in the paragraph above were the main limitation of our study. We designed our study in light of the studies of Rubin et al. (5) and Barry et al. (16). High-risk patients, who may have micrometastasis that could not be detected at the time of operation, were excluded. Thus, the series was homogenized. Finally, 46 of the 92 patients with appropriate specimens for AMACR staining were included in the study. This limitation affecting the patient distribution of the groups may have been determinant in the results of the study.

\section{Conclusions}

The PSA recurrence rate increases with increasing AMAC$C R$ staining intensity. However, no statistically significant relationship was found between the AMACR staining intensity of pathology specimens and postoperative biochemical recurrence (PSA recurrence) in patients undergoing radical prostatectomy for localized prostate cancer. Also, no statistically significant relationship was found between RRP Gleason score, tumor percentile, and AMACR staining intensity, which are essential prognostic parameters for prostate cancer. Further studies are needed to obtain statistically significant results.

\section{Acknowledgements}

Publication: The results of the study were not published in full or in part in form of abstracts.

Contribution: There is not any other contributors who may not be listed as authors.

Conflict of Interest: No conflict of interest was declared by the authors.

Financial Disclosure: The authors declared that this study received no financial support.

\section{Ethics}

Ethics Committee Approval: Retrospective study.
Informed Consent: Retrospective study.

Peer-review: Externally peer-reviewed.

\section{Authorship Contributions}

Concept: M.K., Design: M.K., Data Collection or Processing: M.K., Analysis or Interpretation: E.G., O.Ö., B.A., Literature Search: E.G., S.G., Supervision: B.A., Writing: M.K., O.Ö., S.G., B.A.

\section{References}

1. Schostak M, Miller K, Krause H, Schrader M, Kempkensteffen C, Kollermann J. Kinetic fluorescence reverse transcriptase-polymerase chain reaction for alpha-methylacyl CoA racemase distinguishes prostate cancer from benign lesions. Cancer Detect Prev 2006;30:449-454.

2. Ferdinandusse $S$, Denis $S$, Illst L, Dacremont $G$, Waterham HR, Wanders RJ. Subcellular localization and physiological role of $\alpha$-Methylacyl coenzyme A racemase. J Lipid Res 2000;41:1890-1896.

3. Rhodes DR, Barrette TR, Rubin MA, Ghosh D, Chinnaiyan AM. Metaanalysis of microarrays: interstudy validation of gene expression profiles reveals pathway dysregulation in prostate cancer. Cancer Res 2002;62:4427-4433.

4. Zhou M, Jiang Z, Epstein JI. Expression and diagnostic utility of alpha-methylacyl-CoA-racemase (P504S) in foamy gland and pseudohyperplastic prostate cancer. Am J Surg Pathol 2003;27:772-778.

5. Rubin MA, Bismar TA, Andrén O, et al. Decreased $\alpha-$ Methylacyl CoA racemace expression in localized prostate cancer is associated with an increased rate of biochemical recurrence and cancer-spesific death. Cancer Epidemiol Biomarkers Prev 2005;14:1424-1432.

6. D'Amico AV et al. Biochemical outcome after radical prostatectomy, external beam radiation therapy, or interstitial radiation therapy for clinically localized prostate cancer. JAMA. 1998;280:969-74.

7. Rubin MA, Whittington R, Malkowicz SB, et al. Alpha-Methylacyl coenzyme $A$ racemase as a tissue biomarker for prostate cancer. JAMA 1998;280:969-974.

8. Luo J, Zha S, Gage WR, et al. Alpha-methylacyl-CoA racemase: a new molecular marker for prostate cancer. Cancer Res 2002;62:2220-2226.

9. Adley BP and Yang XJ. Application of alpha-methylacyl coenzyme A racemase immunohistochemistry in the diagnosis of prostate cancer: a review. Anal Quant Cytol Histol 2006;28:1-13.

10. Kumar-Sinha C, Shah RB, Laxman B, et al. Elevated alpha-methylacylCoA racemase enzymatic activity in prostate cancer. Am J Pathol 2004; 16:787-793.

11. Molinié $\mathrm{V}$, Hervé JM, Lugagne PM, et al. Value of new prostate cancer markers: alpha methylacyl CoA racemase (P504S) and p63. Prog Urol 2005;15:611-615.

12. Shapovalova M, Davydova J, Henzler C, et al. Exploiting the transcriptional specificity of the alpha-methylacyl-CoA racemase AMACR promoter for the molecular imaging of prostate cancer. Oncotarget 2018;9:36693-36704.

13. Yang J ,Chen Y, Lu J, et al. Identification and characterization of novel fusion genes in prostate cancer by targeted RNA capture and next-generation sequencing. Acta Biochim Biophys Sin (Shanghai) 2018;50:1166-1172.

14. Etheridge T, Straus J, Ritter MA, Jarrard DF, Huang W. Semen AMACR protein as a novel method for detecting prostate cancer. Urol Oncol 2018;36:532.e1-532.e7.

15. Lloyd MD, Yevglevskis M, Lee GL, Wood PJ, Threadgill MD, Woodman TJ. $\alpha$-Methylacyl-CoA racemase (AMACR): metabolic enzyme, drug metabolizer and cancer marker P504S. Prog Lipid Res 2013;52:220-230.

16. Barry M, Dhillon PK, Stampfer MJ, et al. $\alpha$-Methylacyl-CoA racemase expression and lethal prostate cancer in the Physicians' Health Study and Health Professionals Follow-up Study. Prostate 2012;72:301-306. 